\title{
Bone marrow mesenchymal stromal cells from acute myelogenous leukemiapatients exhibit aberrant gene expression profiles and secreted protein levels
}

Jinxian Wu

Wuhan University Zhongnan Hospital

Xiaoyan Liu

Wuhan University Zhongnan Hospital

Nan Zhang

Wuhan University Zhongnan Hospital

Xinqi Li

Wuhan University Zhongnan Hospital

Hui Shen

Wuhan University Zhongnan Hospital

Li Liu

Wuhan University Zhongnan Hospital

Tingting Huang

Wuhan University Zhongnan Hospital

Lihua Xu

Wuhan University Zhongnan Hospital

Fuling Zhou ( $\square$ zhoufuling@whu.edu.cn )

Wuhan University Zhongnan Hospital https://orcid.org/0000-0003-0982-0382

\section{Research Article}

Keywords: acute myeloid leukemia, mesenchymal stem cells, transcriptome, proteomics, PI3K-akt

Posted Date: November 12th, 2021

DOI: https://doi.org/10.21203/rs.3.rs-1066316/v1

License: (c) (1) This work is licensed under a Creative Commons Attribution 4.0 International License. Read Full License 


\section{Abstract}

Purpose: In hematopoietic malignancies, bone marrow mesenchymal stromal cells (BMSCs) are believed to promote tumor development through cellular and molecular abnormalities. However, there are several discrepancies among BMSCs in patients with acute myeloid leukemia (AML-MSCs) and healthy individuals (HD-MSCs) . Our aim was to analyze the differences in gene and protein levels between AMLMSCs and HD-MSCs, and to explore the role of AML-MSCs in the tumor microenvironment.

Methods: We obtained MSCs from leukemia patients and healthy individuals and identified them by flow cytometry and differentiation. Transcriptome sequencing was performed on MSCs from leukemia patients and healthy individuals and label-free proteomics analysis was conducted on cultured supernatants of MSCs. Finally, we analyzed the results for bioinformatics analysis.

Results: MSCs were preliminarily isolated and identified from AML patients and healthy controls. Through bioinformatics analysis, AML-MSCs and HD-MSCs showed great differences in Gene expression profiles and protein expression profiles of MSCs culture supernatants. Notably, inhibition of PI3K-Akt signal pathway can attenuate chemotherapy resistance of AML cells induced by MSC to AraC.

Conclusions: Together, our findings suggest that Gene expression profiles and secreted protein levels of MSCs culture supernatants differed significantly between AML-MSCs and HD-MSCs, which should greatly facilitate the understanding of the role of MSCs in driving the development of acute myeloid leukemia and exploring new therapeutic strategies in future.

\section{Introduction}

Acute myeloid leukemia (AML) is a group of heterogeneous clonal diseases caused by acquired genetic or epigenetic lesions of hematopoietic stem/progenitor cells(Chaudhury, Morison, Gibson, \& Keeshan, 2015; Shlush et al., 2014; Vedi, Santoro, Dunant, Dick, \& Laurenti, 2016). Most patients still undergo chemotherapy, which is prone to disease recurrence and poor prognosis. Improving the treatment effect and prognosis is an urgent problem to be solved. There is growing evidence to support the importance of abnormal bone marrow microenvironment in the occurrence and development of AML.

Bone marrow microenvironment, also known as bone marrow niche, is composed of mesenchymal stem cells (MSCs), osteoblast (OBC), fat cells, nerve fibers, microvascular networks and extracellular matrix . MSC is an important cell component in the bone marrow microenvironment. It has the potential to differentiate into osteoblasts, chondrocytes and adipocytes under certain conditions. The BMSCs regulate normal and malignant hematopoiesis, but the underlying molecular mechanisms remain poorly defined.

Differences have been reported between the MSCs of leukemia patients and those of healthy donors. Blau et al. found chromosome abnormalities in BMSCs in patients with myelodysplastic syndrome (MDS) and AML, suggesting that BMSCs may be involved in the pathophysiological process of MDS/AML(Blau et al., 2007). In addition, many studies have demonstrated that the contact between 
leukemia cells and cellular components in the bone marrow microenvironment plays an important role in chemoresistance, clonal proliferation of leukemia cells, and disease relapse (Jacamo et al., 2014; Konopleva et al., 2002; Winkler et al., 2012).

Accumulating evidence has shown that MSCs regulate the progression of leukemia largely by secreting distinct sets of cytokines, growth factors, and chemokines (X. Liu et al., 2018; L. Wang et al., 2014; Yehudai-Resheff et al., 2019). Studies have reported that abnormal secretion of MSC-derived cytokines such as transforming growth factor- $\beta 1$ (TGF- $\beta 1$ ), monocyte chemoattractant protein-1 (MCP-1) and prostaglandin E2 (PGE2) and interleukin-6 (IL-6) is associated with AML disease progression (Carter et al., 2019; Geyh et al., 2018; Huang et al., 2015; Sorokina et al., 2016). These factors affect the progression of AML disease by changing the biological characteristics of MSC and regulating signaling pathways in hematopoietic cells.

As to how underlying molecular alterations in BMSCs derived from AML patients (AML-MSCs) contribute to leukemogenesis and chemoresistance remains controversial. The aim of the study was to characterize gene expression profiles and secreted protein expressions of MSCs culture supernatants from AML patients compared to normal BMSCs from healthy donors. In summary, we reported molecular changes in AML BM-MSC, suggesting these altered niche components may benefit future targeted therapies for leukemia.

\section{Materials And Methods}

\section{Cell culture}

MSCs were isolated from fresh BM aspirates with newly diagnosed AML and healthy controls using density gradient centrifugation with Ficoll-Lymphoprep ${ }^{T M}$ (TBD, lot: TBD2013CHU, China). (Supplemental Table S1) after informed written consent following the institution approved protocol in accordance with Declaration of Helsinki requisite ethics approval and informed consent from the participants. Patient diagnosis with AML was based on standard morphological and cytochemical examinations of peripheral blood and marrow smears according to the French-American-British (FAB) and World Health Organization (WHO) criteria (Dohner et al., 2010; Vardiman et al., 2009; Walter et al., 2013). BM-MSCs were expanded in DMEM/F12 (Gibco, USA) supplemented with 10\% fetal bovine serum (Gibco, Australia), $100 \mathrm{U} / \mathrm{ml}$ penicillin, and $100 \mu \mathrm{g} / \mathrm{ml}$ streptomycin at $37^{\circ} \mathrm{C}$ under $5 \% \mathrm{CO} 2$. The medium was replaced every $3 \mathrm{~d}$. The non-adherent cells were removed by completely changing the medium after 3 days, and the adherent cells were continuously cultured.The MSCs from passages 3 were used in all experiments. The MSCs were characterized by surface immunophenotyping using flow cytometry. Differentiation of the MSCs into the adipocyte and osteocyte lineages was verified by morphologic assessment (Supplemental Fig. S1). The AML cell lines of Kasumi- 1 and HL-60 cells were obtained from the cell resource center of Shanghai Institutes for Biological Sciences (Shanghai, China) and maintained in RPMI 1640 media (Gibco, USA) containing $10 \%$ fetal bovine serum (Gibco, Brazil) with $100 \mathrm{U} / \mathrm{mL}$ of penicillin and $100 \mu \mathrm{g} / \mathrm{mL}$ of streptomycin at $37^{\circ} \mathrm{C}$ and $5 \% \mathrm{CO} 2$. 


\section{Drugs}

Cytarabine (AraC) (Actavis Italy) was dissolved in sterile PBS to prepare a $1 \mathrm{mM}$ stock solution and was stored at $-80^{\circ} \mathrm{C}$. LY294002 was purchased from MCE (Shanghai,China).

\section{RNA Library Construction and Sequencing}

Total mRNAs were extracted from AML-MSCs (passage $3, n=5$ ) and HD-MSCs (passage $3, n=5$ ) by using TRIzol reagent (Vazyme, China) and qualified by NanoDrop (Thermo). Preparation of RNA library and transcriptome sequencing was conducted by the Novogene (Tianjin, China). Genes with $p$-value < 0.05 and $\mid \log 2$ (FoldChange) $\mid>0$ were considered as differentially expressed genes (DEGs).

\section{Identification of Differentially Expressed Genes (DEGs)}

\section{DESeq2 R package (1.20.0) was used to screen DEGs between AML-MSCs and HD- MSCs according to the user's guide(Love, Huber, \& Anders, 2014). A heat map and volcano plot of DEGs were drawn by the ggplots package in the R platform.}

\section{Enrichment analysis of differentially expressed genes}

Gene Ontology (GO) enrichment analysis of differentially expressed genes was implemented by the clusterProfiler R package (3.4.4), including biological process (BP), molecular function (MF), and cellular component (CC). We used clusterProfiler $\mathrm{R}$ package to test the statistical enrichment of differential expression genes in KEGG pathways. Adjusted $p$-value less than 0.05 was considered statistically significant(Kanehisa, Furumichi, Tanabe, Sato, \& Morishima, 2017; Yu, Wang, Han, \& He, 2012).

\section{PPI analysis of differentially expressed genes}

To analyze the connection among proteins, DEGs were uploaded to the Search Tool for the Retrieval of Interacting Genes (STRING, https://string-db.org/) (Szklarczyk et al., 2015), which known and predicted Protein-Protein Interactions. The PPI network of DEGs were visualized by Cytoscape software (Shannon et al., 2003). The cytoHubba plugin in Cytoscape was used to identify hub genes (Chin et al., 2014). All parameters of the plugin were left at their default values.

\section{Proteomic analysis of secretded proteins in BMSCs Culture Supernatants}

The MSC medium was replaced with FBS-free DMEM/F12 medium $24 \mathrm{~h}$ before supernatant extraction. After culturing for $24 \mathrm{~h}$, culture supernatant was collected and centrifuged at $1500 \mathrm{rpm}$ for $20 \mathrm{~min}$ to discard nonviable and dead cells and stored at $-80^{\circ} \mathrm{C}$ for subsequent proteomics production assays. The samples collected from MSCs supernatant were analyzed on a Q Exactive mass spectrometer (Thermo Fisher Scientific, Bremen, Germany) by KangChen Bio-tech (Shanghai, China). Raw data were processed with MaxQuant software (version 1.5.6.0). Proteins with differential expression fold change $\geq 1.5, P_{\text {_value }}$ $\leq 0.05$, and unique peptide $\geq 2$ were defined as significantly different, and subsequent $G O$ and KEGG pathway were analyzed. 


\section{Cell apoptosis}

Cell apoptosis was measured using an Annexin V-FITC/PI apoptosis kit (Lianke China) according to the manufacturer's instructions. In total, $1 \times 10^{\wedge} 6 \mathrm{AML}$ cells per well were seeded with MSCs into a 6 -well plate and treated with 1uM Ara and 10uM LY294002. After incubation for $48 \mathrm{~h}$, the cells were harvested and stained with Annexin V-FITC/PI. The apoptotic cells were measured by flow cytometry.

\section{Statistical analysis}

The significance of differences between experimental conditions was determined using the Student $t$ test. A $p$ value $<0.05$ was considered significant.

\section{Results}

\section{AML -MSCs display an altered transcriptional gene expression profile}

To explore transcriptional patterns of AML -MSCs, We performed differential gene expression analysis to compare transcription of AML -MSCs $(n=5)$ to HD-MSCs $(n=5)$. We identified DEGs of RNA-seq between AML-MSCs and HD-MSCs with the criteria of and $P$ value $<0.05$ and $\|$ log2FoldChangel $>0$. Altogether, 668 DEGs were detected, included 349 upregulated genes and 319 downregulated genes (Supplemental Table S2). The volcano plot of the DEGs and the heat map is shown in (Fig. 1A, B). In order to further understand the pathway and process affected by identified DEGs, gene ontology (GO) and Kyoto Encyclopedia of Gene and Genome (KEGG) analyses were respectively performed. By comparing the DEGs of GO analysis, we noticed those upregulated ones in AML-MSCs were involved with cell-cell adhesion via plasma-membrane adhesion molecules, cell-substrate adhesion. Instead, the significantly downregulated genes in AML-MSCs were related to DNA replication, DNA-dependent DNA replication and DNA biosynthetic process (Fig. 1C, D). KEGG pathway enrichment analysis indicated that significant pathways were enrichend in focal adhesion, AGE-RAGE signaling pathway in diabetic complications and PI3K-Akt signaling pathway(Fig. 1E). The top 20 significantly enriched pathways were shown in Table 1.

\section{PPI network construction and module analysis}

To investigate the association of DEGs, protein-protein interactome (PPI) network were conducted through STRING and Cytoscape software. The PPI network constructed from the STRING protein interaction database consisted of 201 nodes and 493 nodes Bars (nodes represent different genes and lines represent interactions between genes) (Fig. 2A). 7 hubgenes COL4A2, COL4A1, COL4A4, COL4A5, COL4A3, ITGA3, ITGA11 were screened by CytoHubba. 7 hub genes with higher degree of connectivity were selected to build the hub gene PPI network (Fig. 2B).

Proteomic analysis screens for proteins that are differentially expressed between AML-MSCs supernatant and HD-MSCs supernatant 
Having illustrated the gene properties of AML-MSCs and HD-MSCs, we attempted to clarify proteins characteristic in AML-MSCs supernatant. To identify secreted protein expressions, we performed labelfree relative quantitative proteomic and bioinformatic analyses. In total, 71 differentially expressed proteins were identified, including 63 up-regulated proteins and 8 down-regulated proteins (fold-change $\geq 1.5$ and $p \leq 0.05$; Fig. $3 A, B$ ). Details regarding altered protein expression are provided in Supplemental Table S3. GO and KEGG enrichment analysis were used to explore the potential function of different secreted proteins. The results indicated that these proteins were mainly enriched in neutrophil mediated immunity, actin cytoskeleton, and actin filament binding(Fig. 3C). Kyoto Encyclopedia of Genes and Genomes (KEGG) pathway analysis revealed that differentially expressed proteins were enriched in Fc gamma R-mediated phagocytosis, shigellosis and regulation of actin cytoskeleton (Fig. 3D).

\section{Resistance of AML-MSCs was reversed by inhibition of PI3K-Akt signaling pathway}

According to our previous results, the PI3K-Akt signaling pathway was abnormally activated in AML MSCs by evaluating KEGG pathway database analysis. What's more, we found that the sensitivity of leukemia cells to AraC decreased after co-culture with MSC. We hypothesized that the PI3K-Akt pathway plays a key role in drug resistance induced by MSC, for the purpose, 10uM LY294002 was added into the co-culture system to inhibit the PI3K-Akt pathway, meanwhile, $2 \mathrm{uM} \mathrm{AraC}$ was added and treated for $48 \mathrm{~h}$, Flow cytometry showed that the drug resistance could be reduced. Collectively, The sensitivity of HL-60 and Kasumi-1 cells to AraC significantly decreased after cocultured with AML-MSCs, whereas the PI3KAkt signal pathway inhibitor LY294002 can significantly increase the sensitivity (Fig. 4).

\section{Discussion}

Bone marrow-derived mesenchymal stromal cells (BMSCs) play a significant role in the BM microenvironment (BME), and abnormalities of these cells may lead to the etiopathogenesis of acute myeloid leukemia (AML). There is increasing evidence that abnormal bone marrow microenvironment can be the initiating factor for the occurrence and maintenance of AML. Bone marrow microenvironment induces AML progression and chemotherapy resistance through self-remodeling(Chen et al., 2016; Cheng et al., 2019; Hanoun et al., 2014). Therefore, understanding the abnormal changes of AML-MSC is helpful to explore the mechanism of action between the abnormal bone marrow microenvironment and AML, and to find new targets for the treatment of AML.

In this study, we identified significant DEGs between AML-MSCs and HD-MSCs. Furthermore, a series of bioinformatics analyses were conducted to identify key genes and pathways. As a result, a total of 668 DEGs were identified, consisting of 349 up-regulated genes and 319 down-regulated genes. GO analyses showed that upregulated and downregulated DEGs were notably abundant in cell-cell adhesion via plasma-membrane adhesion molecules, DNA replication. The KEGG enrichment analysis revealed that DEGs were enriched in pathways included focal adhesion, AGE-RAGE signaling pathway in diabetic complications and PI3K-Akt signaling pathway. Similar results have been described in previous studies, these pathways have been reported to influence the pathogenesis and prognostic development of 
AML (Carter et al., 2017; Darici et al., 2020; Nepstad, Hatfield, Gronningsaeter, \& Reikvam, 2020). By establishing a PPI, seven key genes were found. COL4A1 and COL4A2 are type IV collagen a proteins, and are major components of the basement membrane(Mao, Alavi, Labelle-Dumais, \& Gould, 2015). Studies have shown that COL is involved in the development of liver cancer, cervical cell carcinoma and ovarian cancer(Brown, Brodsky, \& Freiman, 2015; Y. Liu et al., 2020; Vaniotis et al., 2018; Zhou, Li, Peng, Zhou, \& Li, 2020).

Combined with transcriptome and proteomics studies, we found that MRP14 of both were elevated in AML-MSCs. Matrix metalloproteinases (MMP) are a group of proteolytic enzymes that mediate some changes in tumor microenvironment during tumor genesis and development(Kessenbrock, Plaks, \& Werb, 2010). MMP14 is upregulated in a variety of cancers and promotes tumor angiogenesis, inflammation, and tumor progression(Cui, Cai, Ding, \& Gao, 2019; Stawowczyk et al., 2017; Yan et al., 2015). Similarly, there is considerable evidence of abnormal expression of MMP14 in acute leukemia(Lin et al., 2002; Ries, Loher, Zang, Ismair, \& Petrides, 1999; C. Wang, Chen, Li, \& Cen, 2010), but the underlying mechanisms within the bone marrow microenvironment during leukemia cell propagation and invasion have not been fully studied. Wang et al. found that MMP14 was overexpressed in highly invasive leukemia cell lines, and this invasion effect was more obvious when co-cultured with BMSC. Overexpression of MMP14 increased the invasion effect of leukemia cells(C. Wang et al., 2010). Compared with bone marrow mononuclear cells of healthy persons, the increased expression of MMP14 in mononuclear cells of leukemia patients may promote the spread of leukemia cells through local digestion of the ECM barrier, similar to the invasion and metastasis of tumor cells(Ries et al., 1999). We hypothesized that the high expression of MMP14 in AML-MSCs indirectly promotes systemic metastasis of leukemia cells.

The role of molecular changes in bone marrow mesenchymal stromal cells in the pathogenesis of acute myeloid leukemia is unclear. By comparing AML-MSCs and HD-MSCs, Zhang et al. found that the immunophenotype and G-banded karyotype of both were similar, but they had identifiable characteristics in multi-line differentiation, decreased cell viability and multidimensional differences in gene expression profiles(Zhang et al., 2021). However, current studies on AML-MSCs are far from enough. We combined the transcriptome and proteome to confirm the transcriptome differences and secreted proteins of AMLMSCs. It is noteworthy that inhibition of PI3K-Akt pathway can reverse the resistance of MSC to AML cells. Further investigations of these altered MSC may contribute to reveal new tumorigenesis mechanisms and therapeutic strategies. Although our results are a preliminary study of the role and effect of AML-MSCs in the AML bone marrow microenvironment, there are still some limitations. More clinical samples and experimental validation are needed for further validation. In addition, whether AMLMSC can restore the real studies in vivo without the environment of bone marrow matrix remains to be confirmed by research and practice.

Together, our findings suggest that primary BMSCs from AML patients showed different transcription levels and secreted protein levels compared with normal BMSCs from healthy donors. We found that AML-MSCs expressed high MMP14, which may be associated with high aggressiveness of leukemia. Inhibition of PI3K-Akt pathway can reduce the resistance of MSC to AML cells. It will be of interest to 
evaluate the effect of MMP14 secreted by MSC on AML, and to further characterize the effect of abnormal BMSCs on the progression of leukemia.

\section{Declarations}

\section{Contributors}

FLZ conceived and designed the study. JXW and XYL performed the major experiments, analyzed the data, and wrote the manuscript. NZ,XQL and LHX helped to process the specimens and complete the experiment. HS, LL and TTH helped collect specimens and patient information. All authors did the revision of the manuscript and approved the final version.

\section{Funding Statement}

This work was supported by the Natural Science Foundation of China (NSFC) programme [grant number 81770179], Zhongnan Hospital of Wuhan University Science, Technology and Innovation Cultivation Fund [grant number ZNLH201902], and the Natural Science Foundation of China (NSFC) programme [grant number 81900116].

\section{Conflicts of Interest}

All authors declare that the the research was conducted in the absence of any commercial or financial relationships that could be construed as a potential conflict of interest.

\section{References}

Blau, O., Hofmann, W. K., Baldus, C. D., Thiel, G., Serbent, V., Schumann, E., .. Blau, I. W. (2007). Chromosomal aberrations in bone marrow mesenchymal stroma cells from patients with myelodysplastic syndrome and acute myeloblastic leukemia. Exp Hematol, 35(2), 221-229. doi:10.1016/j.exphem.2006.10.012

Brown, C. W., Brodsky, A. S., \& Freiman, R. N. (2015). Notch3 overexpression promotes anoikis resistance in epithelial ovarian cancer via upregulation of COL4A2. Mol Cancer Res, 13(1), 78-85. doi:10.1158/15417786.MCR-14-0334

Carter, B. Z., Mak, P. Y., Wang, X., Tao, W., Ruvolo, V., Mak, D., . . Andreeff, M. (2019). An ARC-Regulated IL1beta/Cox-2/PGE2/beta-Catenin/ARC Circuit Controls Leukemia-Microenvironment Interactions and Confers Drug Resistance in AML. Cancer Res, 79(6), 1165-1177. doi:10.1158/0008-5472.CAN-18-0921

Carter, B. Z., Mak, P. Y., Wang, X., Yang, H., Garcia-Manero, G., Mak, D. H., . . Andreeff, M. (2017). Focal Adhesion Kinase as a Potential Target in AML and MDS. Mol Cancer Ther, 16(6), 1133-1144. doi:10.1158/1535-7163.MCT-16-0719 
Chaudhury, S. S., Morison, J. K., Gibson, B. E., \& Keeshan, K. (2015). Insights into cell ontogeny, age, and acute myeloid leukemia. Exp Hematol, 43(9), 745-755. doi:10.1016/j.exphem.2015.05.008

Chen, S., Zambetti, N. A., Bindels, E. M., Kenswill, K., Mylona, A. M., Adisty, N. M., . . Raaijmakers, M. H. (2016). Massive parallel RNA sequencing of highly purified mesenchymal elements in low-risk MDS reveals tissue-context-dependent activation of inflammatory programs. Leukemia, 30(9), 1938-1942. doi:10.1038/leu.2016.91

Cheng, J., Li, Y., Liu, S., Jiang, Y., Ma, J., Wan, L., . . Pang, T. (2019). CXCL8 derived from mesenchymal stromal cells supports survival and proliferation of acute myeloid leukemia cells through the PI3K/AKT pathway. FASEB J, 33(4), 4755-4764. doi:10.1096/fj.201801931R

Chin, C. H., Chen, S. H., Wu, H. H., Ho, C. W., Ko, M. T., \& Lin, C. Y. (2014). cytoHubba: identifying hub objects and sub-networks from complex interactome. BMC Syst Biol, 8 Suppl 4, S11. doi:10.1186/17520509-8-S4-S11

Cui, G., Cai, F., Ding, Z., \& Gao, L. (2019). MMP14 predicts a poor prognosis in patients with colorectal cancer. Hum Pathol, 83, 36-42. doi:10.1016/j.humpath.2018.03.030

Darici, S., Alkhaldi, H., Horne, G., Jorgensen, H. G., Marmiroli, S., \& Huang, X. (2020). Targeting PI3K/Akt/mTOR in AML: Rationale and Clinical Evidence. J Clin Med, 9(9). doi:10.3390/jcm9092934

Dohner, H., Estey, E. H., Amadori, S., Appelbaum, F. R., Buchner, T., Burnett, A. K., . . European, LeukemiaNet. (2010). Diagnosis and management of acute myeloid leukemia in adults:

recommendations from an international expert panel, on behalf of the European LeukemiaNet. Blood, 115(3), 453-474. doi:10.1182/blood-2009-07-235358

Geyh, S., Rodriguez-Paredes, M., Jager, P., Koch, A., Bormann, F., Gutekunst, J., . . S Schroeder, T. (2018). Transforming growth factor beta1-mediated functional inhibition of mesenchymal stromal cells in myelodysplastic syndromes and acute myeloid leukemia. Haematologica, 103(9), 1462-1471. doi:10.3324/haematol.2017.186734

Hanoun, M., Zhang, D., Mizoguchi, T., Pinho, S., Pierce, H., Kunisaki, Y., . . Frenette, P. S. (2014). Acute myelogenous leukemia-induced sympathetic neuropathy promotes malignancy in an altered hematopoietic stem cell niche. Cell Stem Cell, 15(3), 365-375. doi:10.1016/j.stem.2014.06.020

Huang, J. C., Basu, S. K., Zhao, X., Chien, S., Fang, M., Oehler, V. G., . . Becker, P. S. (2015). Mesenchymal stromal cells derived from acute myeloid leukemia bone marrow exhibit aberrant cytogenetics and cytokine elaboration. Blood Cancer J, 5, e302. doi:10.1038/bcj.2015.17

Jacamo, R., Chen, Y., Wang, Z., Ma, W., Zhang, M., Spaeth, E. L., . . Andreeff, M. (2014). Reciprocal leukemia-stroma VCAM-1/VLA-4-dependent activation of NF-kappaB mediates chemoresistance. Blood, 123(17), 2691-2702. doi:10.1182/blood-2013-06-511527 
Kanehisa, M., Furumichi, M., Tanabe, M., Sato, Y., \& Morishima, K. (2017). KEGG: new perspectives on genomes, pathways, diseases and drugs. Nucleic Acids Res, 45(D1), D353-D361.

doi:10.1093/nar/gkw1092

Kessenbrock, K., Plaks, V., \& Werb, Z. (2010). Matrix metalloproteinases: regulators of the tumor microenvironment. Cell, 141(1), 52-67. doi:10.1016/j.cell.2010.03.015

Konopleva, M., Konoplev, S., Hu, W., Zaritskey, A. Y., Afanasiev, B. V., \& Andreeff, M. (2002). Stromal cells prevent apoptosis of AML cells by up-regulation of anti-apoptotic proteins. Leukemia, 16(9), 1713-1724. doi:10.1038/sj.leu.2402608

Lin, L. I., Lin, D. T., Chang, C. J., Lee, C. Y., Tang, J. L., \& Tien, H. F. (2002). Marrow matrix metalloproteinases (MMPs) and tissue inhibitors of MMP in acute leukaemia: potential role of MMP-9 as a surrogate marker to monitor leukaemic status in patients with acute myelogenous leukaemia. $\mathrm{Br} \mathrm{J}$ Haematol, 117(4), 835-841. doi:10.1046/j.1365-2141.2002.03510.x

Liu, X., Ma, Y., Li, R., Guo, D., Wang, N., Zhao, Y., . . . Ma, X. (2018). Niche TWIST1 is critical for maintaining normal hematopoiesis and impeding leukemia progression. Haematologica, 103(12), 1969-1979. doi:10.3324/haematol.2018.190652

Liu, Y., Zhang, J., Chen, Y., Sohel, H., Ke, X., Chen, J., \& Li, Y. X. (2020). The correlation and role analysis of COL4A1 and COL4A2 in hepatocarcinogenesis. Aging (Albany NY), 12(1), 204-223.

doi:10.18632/aging. 102610

Love, M. I., Huber, W., \& Anders, S. (2014). Moderated estimation of fold change and dispersion for RNAseq data with DESeq2. Genome Biol, 15(12), 550. doi:10.1186/s13059-014-0550-8

Mao, M., Alavi, M. V., Labelle-Dumais, C., \& Gould, D. B. (2015). Type IV Collagens and Basement Membrane Diseases: Cell Biology and Pathogenic Mechanisms. Curr Top Membr, 76, 61-116. doi:10.1016/bs.ctm.2015.09.002

Nepstad, I., Hatfield, K. J., Gronningsaeter, I. S., \& Reikvam, H. (2020). The PI3K-Akt-mTOR Signaling Pathway in Human Acute Myeloid Leukemia (AML) Cells. Int J Mol Sci, 21(8). doi:10.3390/ijms21082907 Ries, C., Loher, F., Zang, C., Ismair, M. G., \& Petrides, P. E. (1999). Matrix metalloproteinase production by bone marrow mononuclear cells from normal individuals and patients with acute and chronic myeloid leukemia or myelodysplastic syndromes. Clin Cancer Res, 5(5), 1115-1124.

Shannon, P., Markiel, A., Ozier, O., Baliga, N. S., Wang, J. T., Ramage, D., . . Ideker, T. (2003). Cytoscape: a software environment for integrated models of biomolecular interaction networks. Genome Res, 13(11), 2498-2504. doi:10.1101/gr.1239303

Shlush, L. I., Zandi, S., Mitchell, A., Chen, W. C., Brandwein, J. M., Gupta, V., . . Dick, J. E. (2014). Identification of pre-leukaemic haematopoietic stem cells in acute leukaemia. Nature, 506(7488), 328- 
Sorokina, T., Shipounova, I., Bigildeev, A., Petinati, N., Drize, N., Turkina, A., . . Savchenko, V. (2016). The ability of multipotent mesenchymal stromal cells from the bone marrow of patients with leukemia to maintain normal hematopoietic progenitor cells. Eur J Haematol, 97(3), 245-252. doi:10.1111/ejh.12713

Stawowczyk, M., Wellenstein, M. D., Lee, S. B., Yomtoubian, S., Durrans, A., Choi, H., . . Mittal, V. (2017). Matrix Metalloproteinase 14 promotes lung cancer by cleavage of Heparin-Binding EGF-like Growth Factor. Neoplasia, 19(2), 55-64. doi:10.1016/j.neo.2016.11.005

Szklarczyk, D., Franceschini, A., Wyder, S., Forslund, K., Heller, D., Huerta-Cepas, J., . . von Mering, C. (2015). STRING v10: protein-protein interaction networks, integrated over the tree of life. Nucleic Acids Res, 43(Database issue), D447-452. doi:10.1093/nar/gku1003

Vaniotis, G., Rayes, R. F., Qi, S., Milette, S., Wang, N., Perrino, S., . . Brodt, P. (2018). Collagen IV-conveyed signals can regulate chemokine production and promote liver metastasis. Oncogene, 37(28), 3790-3805. doi:10.1038/s41388-018-0242-z

Vardiman, J. W., Thiele, J., Arber, D. A., Brunning, R. D., Borowitz, M. J., Porwit, A., . . Bloomfield, C. D. (2009). The 2008 revision of the World Health Organization (WHO) classification of myeloid neoplasms and acute leukemia: rationale and important changes. Blood, 114(5), 937-951. doi:10.1182/blood-200903-209262

Vedi, A., Santoro, A., Dunant, C. F., Dick, J. E., \& Laurenti, E. (2016). Molecular landscapes of human hematopoietic stem cells in health and leukemia. Ann NY Acad Sci, 1370(1), 5-14.

doi:10.1111/nyas.12981

Walter, R. B., Othus, M., Burnett, A. K., Lowenberg, B., Kantarjian, H. M., Ossenkoppele, G. J., . . Estey, E. H. (2013). Significance of FAB subclassification of "acute myeloid leukemia, NOS" in the 2008 WHO classification: analysis of 5848 newly diagnosed patients. Blood, 121(13), 2424-2431. doi:10.1182/blood2012-10-462440

Wang, C., Chen, Z., Li, Z., \& Cen, J. (2010). The essential roles of matrix metalloproteinase-2, membrane type 1 metalloproteinase and tissue inhibitor of metalloproteinase-2 in the invasive capacity of acute monocytic leukemia SHI-1 cells. Leuk Res, 34(8), 1083-1090. doi:10.1016/j.leukres.2010.01.016

Wang, L., Zhang, H., Rodriguez, S., Cao, L., Parish, J., Mumaw, C., . . Carlesso, N. (2014). Notch-dependent repression of miR-155 in the bone marrow niche regulates hematopoiesis in an NF-kappaB-dependent manner. Cell Stem Cell, 15(1), 51-65. doi:10.1016/j.stem.2014.04.021

Winkler, I. G., Barbier, V., Nowlan, B., Jacobsen, R. N., Forristal, C. E., Patton, J. T., . . Levesque, J. P. (2012). Vascular niche E-selectin regulates hematopoietic stem cell dormancy, self renewal and chemoresistance. Nat Med, 18(11), 1651-1657. doi:10.1038/nm.2969 
Yan, T., Lin, Z., Jiang, J., Lu, S., Chen, M., Que, H., .. Zheng, Q. (2015). MMP14 regulates cell migration and invasion through epithelial-mesenchymal transition in nasopharyngeal carcinoma. Am J Transl Res, $7(5), 950-958$.

Yehudai-Resheff, S., Attias-Turgeman, S., Sabbah, R., Gabay, T., Musallam, R., Fridman-Dror, A., \& Zuckerman, T. (2019). Abnormal morphological and functional nature of bone marrow stromal cells provides preferential support for survival of acute myeloid leukemia cells. Int J Cancer, 144(9), 22792289. doi:10.1002/ijc.32063

Yu, G., Wang, L. G., Han, Y., \& He, Q. Y. (2012). clusterProfiler: an R package for comparing biological themes among gene clusters. OMICS, 16(5), 284-287. doi:10.1089/omi.2011.0118

Zhang, L., Chi, Y., Wei, Y., Zhang, W., Wang, F., Zhang, L., . . Han, Z. (2021). Bone marrow-derived mesenchymal stem/stromal cells in patients with acute myeloid leukemia reveal transcriptome alterations and deficiency in cellular vitality. Stem Cell Res Ther, 12(1), 365. doi:10.1186/s13287-02102444-0

Zhou, C., Li, C., Peng, S., Zhou, L., \& Li, H. (2020). Comprehensive Analysis of the Relationships Between Tumor Mutation Burden With Immune Infiltrates in Cervical Cell Carcinoma. Front Mol Biosci, 7, 582911. doi:10.3389/fmolb.2020.582911

\section{Tables}

Due to technical limitations, Table 1 is only available as a download in the Supplemental Files section.

\section{Figures}


A

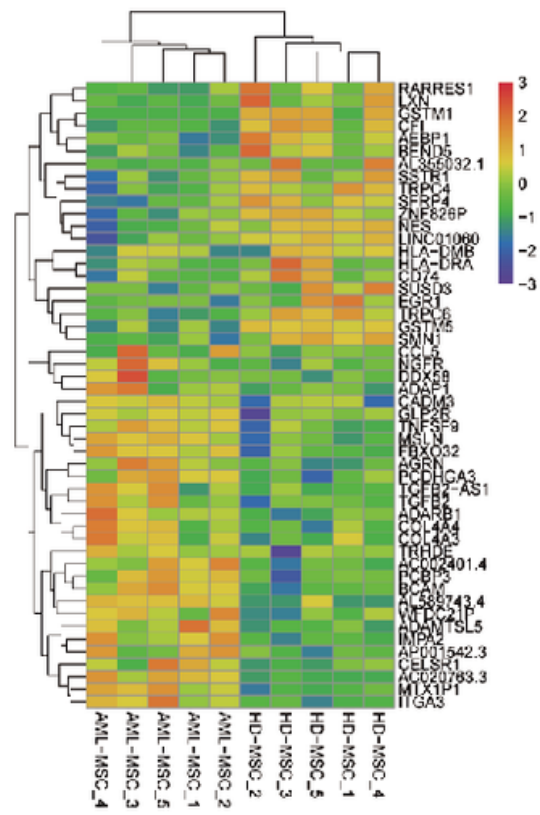

C

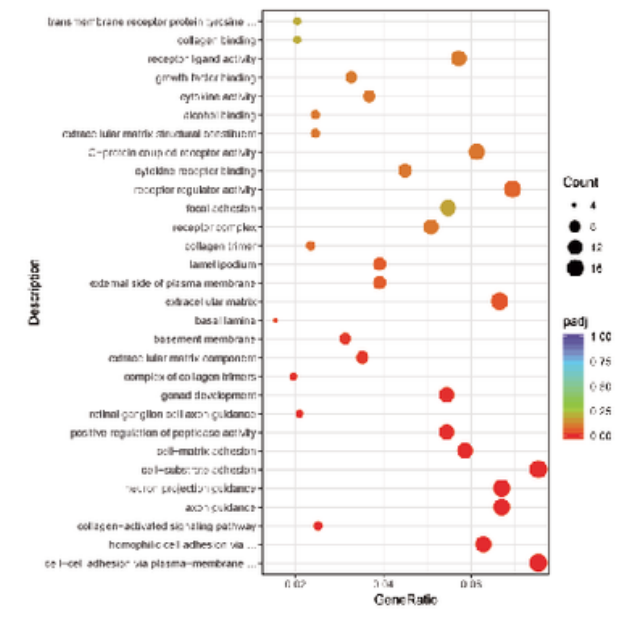

B

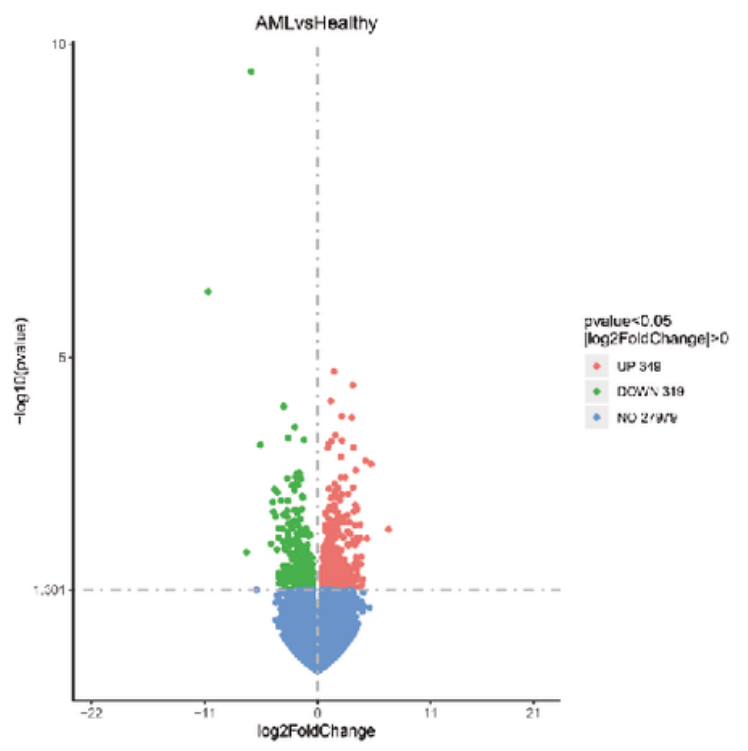

$E$

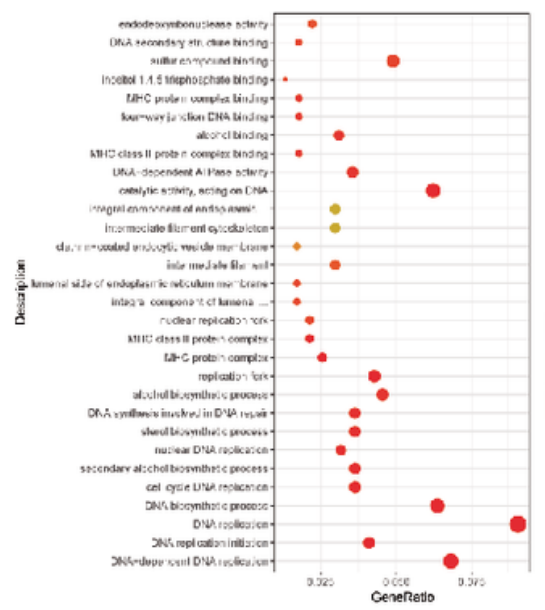

Figure 1

Differential expression genes of AML-MSCs and HD-MSCs (A) Heat map for the diferentially expressed genes based on AML-MSCs and HD-MSCs. (B) Volcano plot of genes, red dots represent upregulated and green dots represent downregulated. Gene ontology of upregulated genes (C) and downregulated genes(D) in AML-MSCs compared with HD-MSCs. (E)The enriched KEGG pathway in AML-MSCs compared with those in HD-MSCs . 


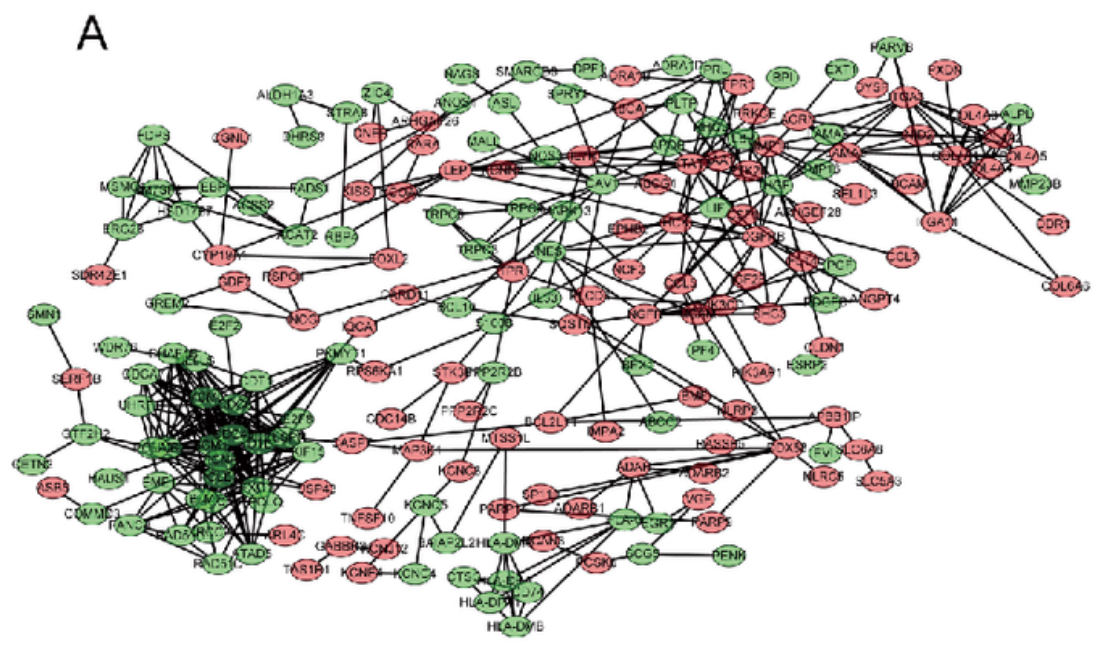

B

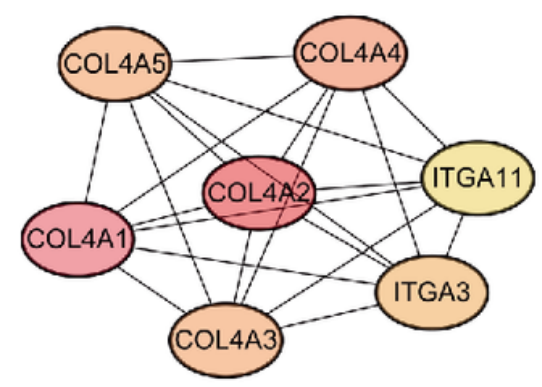

Figure 2

The protein-protein interactome (PPI) network and hub genes. (A) PPI network of differentially expressed genes. Red nodes mean upregulated differentially expressed genes, and green nodes mean downregulated differentially expressed genes. (B) The PPI network the top 7 hub genes. 

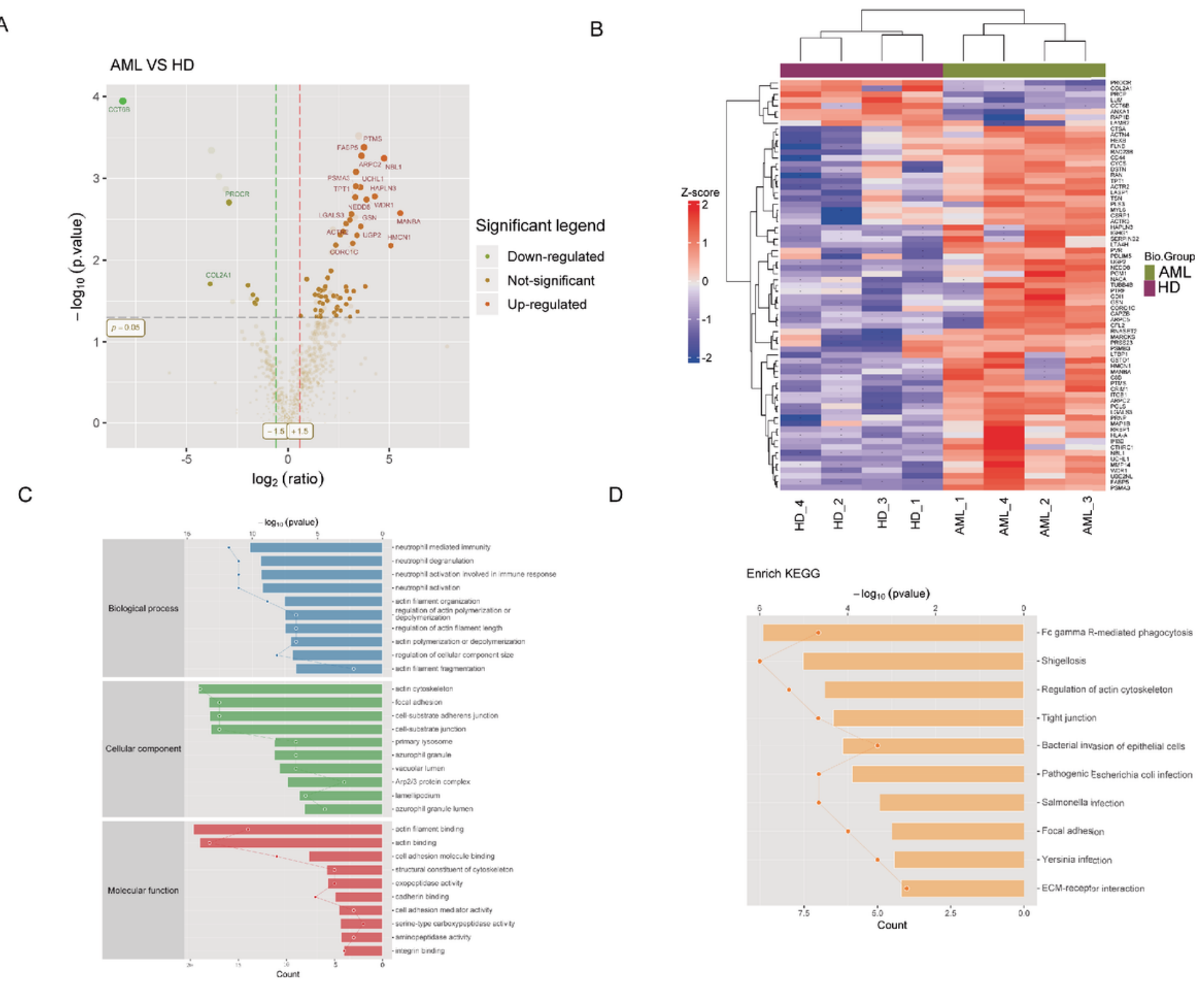

Figure 3

Differential protein expressions in the cellular supernatant of AML-MSCs compared with HD-MSCs.

(A)Volcano plots reflecting number, significance and reliability of differentially expressed proteins in AMLMSCs compared with those in HD-MSCs. The red dots indicate upregulation and green dots indicate downregulation of proteins. (B)A heatmap representation of a total of 71differentially expressed analytes measured by labelfree assay using the cellular supernatant obtained from AML-MSCs $(n=4)$ and HDMSCs ( $n=4)$. Heatmap rows and columns were hierarchically clustered (unsupervised) within the groups. (C) Gene ontology (GO) analysis of proteins. (D) Pathway analysis of differentially expressed proteins. 

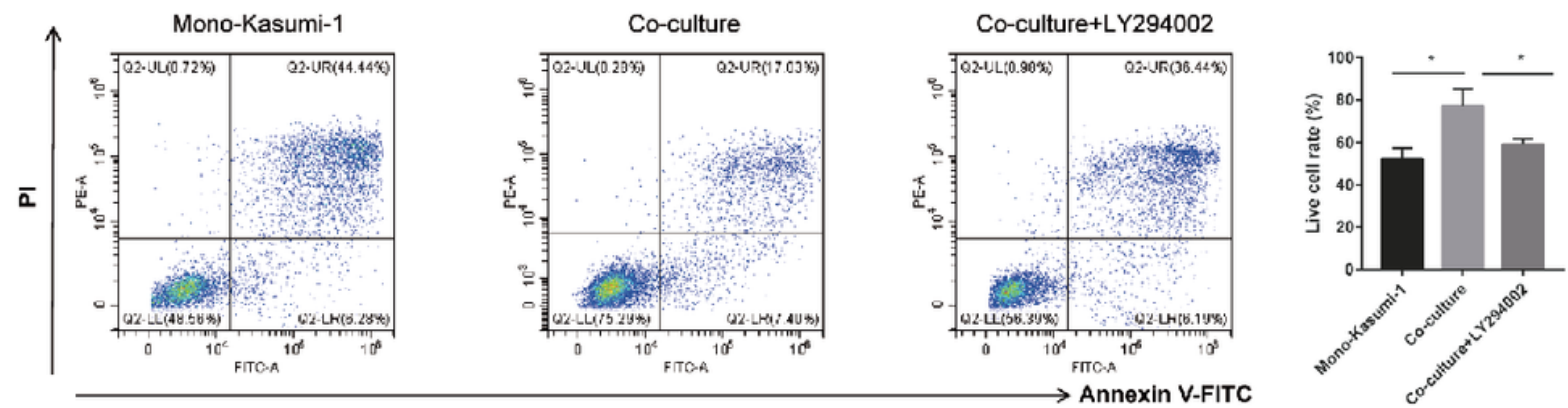

- Mono-Kasumi-1

- Cocuiture

- Co-culture+LY294002
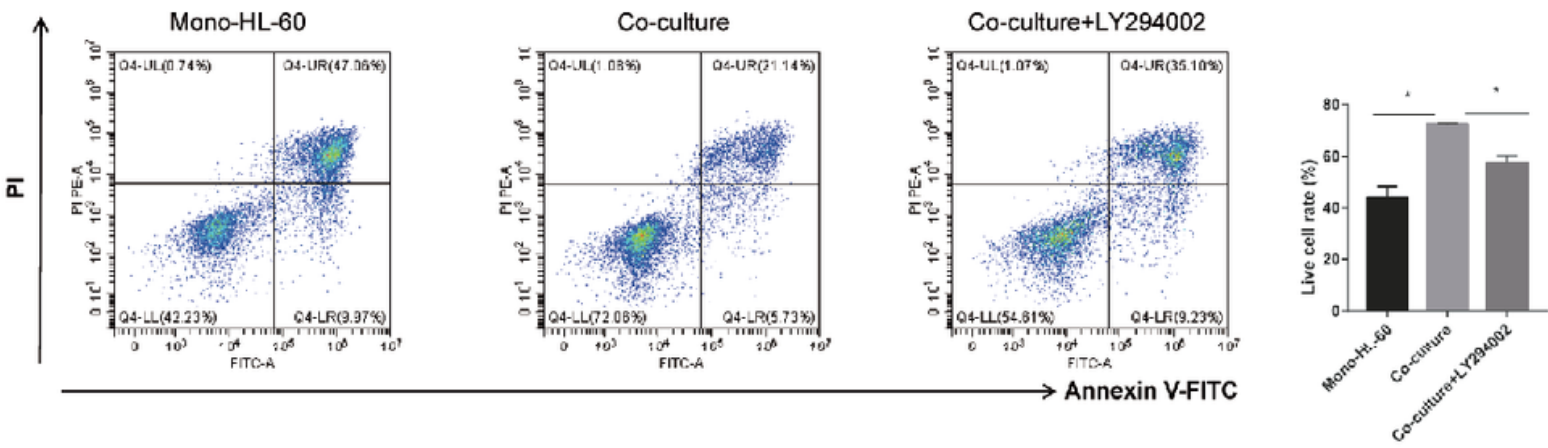

- Mono-HL-60 Co-culure - Co-culure+LY29400

Figure 4

LY294002 enhanced the anti-leukemia effect of AraC on Co-HL-60 and Co-Kasumi-1 cells by inhibiting the activated PI3K/Akt signaling pathway. Coculture with MSCs significantly decreased drug sensitivity of HL-60 cells and Ksaumi- 1 cells, whereas LY294002 increased the sensitivity. AML cells $\left(1 \times 10^{\wedge} 6 / \mathrm{ml}\right)$ were treated with AraC or LY294002 for $48 \mathrm{~h}$ after being treated alone or co-cultured with MSCs. Apoptosis was tested by flow cytometry. The experiments were repeated three times. The results are presented as mean \pm SD. * $p<0.05$.

\section{Supplementary Files}

This is a list of supplementary files associated with this preprint. Click to download.

- Table1.docx

- Figs1.tif

- TableS1.docx

- Tables2.xlsx

- Tables3.xls 